\title{
Numerical Inclusion of Optimum Point for Linear Programming
}

\author{
Shin'ichi Oishi ${ }^{1,2}$ and Kunio Tanabe ${ }^{1}$ \\ Department of Applied Mathematics, Faculty of Science and Engineering, Waseda University, \\ Tokyo 169-8555, Japan ${ }^{1}$ and CREST, JST, Japan ${ }^{2}$ \\ E-mailoishi@waseda.jp,tanabe.kunio@waseda.jp
}

Received August 31, 2008, Accepted October 6, 2008 (INVITED PAPER)

\begin{abstract}
This paper concerns with the following linear programming problem:

Maximize $c^{t} x$, subject to $A x \leqq b$ and $x \geqq 0$,

where $A \in \mathbb{F}^{m \times n}, b \in \mathbb{F}^{m}$ and $c, x \in \mathbb{F}^{n}$. Here, $\mathbb{F}$ is a set of floating point numbers.

The aim of this paper is to propose a numerical method of including an optimum point of this linear programming problem provided that a good approximation of an optimum point is given. The proposed method is base on Kantorovich's theorem and the continuous Newton method. Kantorovich's theorem is used for proving the existence of a solution for complimentarity equation and the continuous Newton method is used to prove feasibility of that solution. Numerical examples show that a computational cost to include optimum point is about 4 times than that for getting an approximate optimum solution.
\end{abstract}

Keywords numerical verification, continuous Newton method, Kantorovich's theorem Research Activity Group Quality of Computations

\section{Introduction}

In this paper, we are concerned with the following linear programming problem:

Maximize $c^{t} x$, subject to $A x \leqq b$ and $x \geqq 0$,

where $A \in \mathbb{F}^{m \times n}, b \in \mathbb{F}^{m}$ and $c, x \in \mathbb{F}^{n}$. Here, $\mathbb{F}$ is a set of floating point numbers. The superscript $t$ indicates the transposition. The aim of this paper is to propose a numerical method of including an optimum point of this linear programming problem provided that a good approximation of an optimum point is given.

Let $x_{f}$ be a feasible point of the primal problem (1), i.e., $x_{f}$ be a point satisfying

$$
A x_{f} \leqq b \text { and } x_{f} \geqq 0 .
$$

It is clear that $c^{t} x_{f}$ becomes a lower bound of the optimum value. A dual problem for (1) is given by

Minimize $b^{t} y$, subject to $A^{t} y \geqq c$ and $y \geqq 0$,

where $y \in \mathbb{F}^{m}$. Let $y_{f}$ be a feasible point of the dual problem (3), i.e., $y_{f}$ be a point satisfying

$$
A^{t} y_{f} \geqq c \text { and } y_{f} \geqq 0 \text {. }
$$

It is clear that $b^{t} y_{f}$ becomes an upper bound of the optimum value. Thus, an inclusion of the optimum value $v^{*}$ is given by

$$
v^{*} \in\left[c^{t} x_{f}, b^{t} y_{f}\right]
$$

provided that feasible points $x_{f}$ and $y_{f}$ can be found. The duality theorem asserts that in principle the width of the interval $\left[c^{t} x_{f}, b^{t} y_{f}\right]$ can make as small as desired. This argument, which is rather well known (cf. Ref. [1]), gives a method of numerical inclusion of the optimum value.

This paper is concerned with a problem of including numerically an optimum point of (1). For the purpose, we shall consider the following complimentarity problem

$$
f(z)=\left(\begin{array}{c}
x\left(A^{t} y-c\right) \\
y(b-A x)
\end{array}\right)=0 \in \mathbb{R}^{n+m}
$$

subject to

$$
x \geqq 0, y \geqq 0, b-A x \geqq 0 \text { and } A^{t} y-c \geqq 0,
$$

where $z=\left(x^{t}, y^{t}\right)^{t}$. Here, for two vectors $u, v$ with the same dimension, $u v$ denotes a vector of the same dimension with components $u_{i} v_{i}$. To solve (6) subject to (7) is equivalent to solve the primal and dual problem (1) and (3). Tanabe [2] has applied Kantorovich's theorem to (6) to estimate error in an approximate solution. However, the solution proved to be included by this approach is not guaranteed to satisfy the feasibility condition (7). This paper resolves this difficulty by introducing a continuous Newton method. Namely, this paper proposes a method, in which Kantorovich's theorem is used for proving the existence of a solution for complimentarity equation and the continuous Newton method is exploited to prove feasibility of that solution. Since Kantorovich's theorem for the Newton method is used, non-degeneracy of the optimum solution is required for our analysis. Degenerate cases will be considered in a separate paper. 


\section{Verification Method}

The center path of (6) (cf. for instance Refs. [3-5]) is defined by

$$
f(z)=\left(\begin{array}{c}
x\left(A^{t} y-c\right) \\
y(b-A x)
\end{array}\right)=\gamma e,
$$

where $e \in \mathbb{R}^{m+n}$ with all elements being 1 . The constant $\gamma$ is defined by

$$
\gamma=\frac{\|f(z)\|_{1}}{m+n}=\frac{\left(b^{t} y-c^{t} x\right)}{m+n}
$$

provided that $z$ is a feasible point. Namely, if $z$ is a feasible point, $\gamma$ is the duality gap of the problem divided by $m+n$. This fact is pointed out in Refs. [4,5].

The Fréchet derivative $f^{\prime}(z)$ is given by

$$
f^{\prime}(z)=\left(\begin{array}{cc}
{\left[A^{t} y-c\right]} & {[x] A^{t}} \\
-[y] A & {[b-A x]}
\end{array}\right),
$$

where for a vector $x=\left(x_{1}, x_{2}, \cdots, x_{n}\right)^{t},[x]$ denotes $\operatorname{diag}\left(x_{1}, x_{2}, \cdots, x_{n}\right)$. At a given approximate optimum point $z$, the Newton direction $d_{n}$ and the centered direction $d_{c}$ are defined by

$$
f^{\prime}(z) d_{n}=-\left(\begin{array}{c}
x\left(A^{t} y-c\right) \\
y(b-A x)
\end{array}\right)
$$

and

$$
f^{\prime}(z) d_{c}=-\left(\begin{array}{c}
x\left(A^{t} y-c\right) \\
y(b-A x)
\end{array}\right)+\gamma e,
$$

respectively.

In the method we shall propose the following verification procedures. First, an interior point of (6) is searched for a searching direction, which is a linear combination of $d_{n}$ and $d_{c}$, based on the guiding cone method or the penelalized norm method $[4,5]$. Here, we assume that we can find an interior point $z$, which is a good approximation of an optimum point. Then, the second step of our method is to check conditions of the following theorem at the point $z$ :

Theorem 1 Let $z \in \mathbb{R}^{m+n}$ be an interior point, namely a point satisfying (7) with inequality condition:

$$
x>0, y>0, b-A x>0 \text { and } A^{t} y-c>0 .
$$

Let further constants $\alpha$ and $\omega$ be defined by the inequalities $\alpha \geqq\left\|f^{\prime}(z)^{-1}\right\|_{\infty}\|f(z)\|_{\infty}$ and $\omega \geqq$ $2 \max \left(\|A\|_{\infty},\|A\|_{1}\right)\left\|f^{\prime}(z)^{-1}\right\|_{\infty}$, respectively. If

$$
\alpha \omega \leqq \frac{1}{4},
$$

there exists an optimal point $z^{*}=\left(x^{* t}, y^{* t}\right)^{t} \in B(z, \rho)=$ $\left\{z^{\prime} \in \mathbb{R}^{m+n} \mid\left\|z^{\prime}-z\right\|_{\infty} \leqq \rho\right\}$, which is a point satisfying (6) and (7), where

$$
\rho=\frac{1-\sqrt{1-3 \alpha \omega}}{\omega} .
$$

The optimum point $z^{*}$ is unique in $B(z, \rho)$.

We note that the half assertion of Theorem 1 can be derived from the following Kantorovich theorem applied to the nonlinear equation (6):

Theorem 2 (Kantorovich's Theorem for (6)) Let $f$ be defined by (6). We assume that the Fréchet derivative $f^{\prime}(z)$ is nonsingular and satisfies the inequality

$$
\alpha^{\prime} \geqq\left\|f^{\prime}(z)^{-1} f(z)\right\|_{\infty}
$$

for a certain positive $\alpha^{\prime}$. Furthermore, we assume that $f$ satisfies

$$
\begin{aligned}
& \left\|f^{\prime}(z)^{-1}\left(f^{\prime}\left(z^{\prime}\right)-f^{\prime}\left(z^{\prime \prime}\right)\right)\right\|_{\infty} \\
\leqq & \omega^{\prime}\left\|z^{\prime}-z^{\prime \prime}\right\|_{\infty} \text { for } \forall z^{\prime}, z^{\prime \prime} \in \mathbb{R}^{m+n}
\end{aligned}
$$

with a certain positive constant $\omega^{\prime}$. If

$$
\alpha^{\prime} \omega^{\prime} \leqq \frac{1}{2}
$$

and

$$
\rho^{\prime}=\frac{1-\sqrt{1-2 \alpha^{\prime} \omega^{\prime}}}{\omega^{\prime}}
$$

there exists a point $z^{*}=\left(x^{* t}, y^{* t}\right)^{t} \in B\left(z, \rho^{\prime}\right)$ satisfying (6). The solution $z^{*}$ of $(6)$ is unique in $B\left(z, \rho^{\prime}\right)$.

Proof of Theorem 1 We assume that the conditions of Theorem 1 is satisfied.

In the first place, we shall show that the conditions of Theorem 2 are satisfied. We note that $f$ is defined on $\mathbb{R}^{m+n}$. If we put $\alpha^{\prime}=1.5 \alpha$, then

$$
\begin{aligned}
\left\|f^{\prime}(z)^{-1} f(z)\right\|_{\infty} & \leqq\left\|f^{\prime}(z)^{-1}\right\|_{\infty}\|f(z)\|_{\infty} \\
& \leqq \alpha<\alpha^{\prime} .
\end{aligned}
$$

It is further noted that for any $z^{\prime}, z^{\prime \prime} \in \mathbb{R}^{m+n}$

$$
\begin{aligned}
& f^{\prime}\left(z^{\prime}\right)-f^{\prime}\left(z^{\prime \prime}\right) \\
= & \left(\begin{array}{cc}
{\left[A^{t}\left(y^{\prime}-y^{\prime \prime}\right)\right]} & {\left[x^{\prime}-x^{\prime \prime}\right] A^{t}} \\
-\left[y^{\prime}-y^{\prime \prime}\right] A & {\left[-A\left(x^{\prime}-x^{\prime \prime}\right)\right]}
\end{array}\right) .
\end{aligned}
$$

Let $e_{k}=(1,1, \cdots, 1)^{t} \in \mathbb{R}^{k}$ and $I_{k}$ be the identity matrix in $\mathbb{R}^{k}$. Then, from (21), we have the following elementwise inequality:

$$
\begin{aligned}
& \left|f^{\prime}\left(z^{\prime}\right)-f^{\prime}\left(z^{\prime \prime}\right)\right| \\
\leqq & \left\|z^{\prime}-z^{\prime \prime}\right\|_{\infty}\left(\begin{array}{cc}
{\left[|A|^{t} e_{m}\right]} & {\left[e_{n}\right]|A|^{t}} \\
{\left[e_{m}\right]|A|} & {\left[|A| e_{n}\right]}
\end{array}\right) \\
\leqq & \left\|z^{\prime}-z^{\prime \prime}\right\|_{\infty}\left(\begin{array}{cc}
\|A\|_{1} I_{n} & |A|^{t} \\
|A| & \|A\|_{\infty} I_{m}
\end{array}\right)
\end{aligned}
$$

which implies

$$
\begin{aligned}
& \left\|f^{\prime}\left(z^{\prime}\right)-f^{\prime}\left(z^{\prime \prime}\right)\right\|_{\infty} \\
\leqq & 2 \max \left(\|A\|_{\infty},\|A\|_{1}\right)\left\|z^{\prime}-z^{\prime \prime}\right\|_{\infty} .
\end{aligned}
$$

Here, for $x=\left(x_{1}, x_{2}, \cdots, x_{k}\right)^{t}, y=\left(y_{1}, y_{2}, \cdots, y_{k}\right)^{t} \in$ $\mathbb{R}^{k}$

$$
|x|=\left(\left|x_{1}\right|,\left|x_{2}\right|, \cdots,\left|x_{k}\right|\right)^{t}
$$

and

$$
x \leqq y \Longleftrightarrow x_{i} \leqq y_{i}, \quad(i=1,2, \cdots, k) .
$$

Hence, we can use $\omega$ in Theorem 1 as $\omega^{\prime}$ in Theorem 2. If we put $\alpha^{\prime}=1.5 \alpha$ and $\omega^{\prime}=\omega$, we have

$$
\alpha^{\prime} \omega^{\prime}=1.5 \alpha \omega \leqq 3 / 8<1 / 2 \text {. }
$$

Further, $\rho^{\prime}$ coincides with $\rho$. Thus, from Kantorovich's theorem (Theorem 2) it is seen that there exists a solution $z^{*}=\left(x^{* t}, y^{* t}\right)^{t} \in B(z, \rho)$ satisfying (6). Kan- 
torovich's theorem states also that $z^{*}$ is unique solution of (6) in the closed ball $B(z, \rho)$.

Next, we show that $z^{*}$ is feasible, i.e., it satisfies the inequality conditions (7). Let us consider a solution curve of the following continuous Newton method starting from a given interior point $z$ :

$$
\frac{d z(t)}{d t}=-f^{\prime}(z(t))^{-1} f(z(t)) \text { with } z(0)=z
$$

The elementary theory for differential equations such as the Picard-Lindelöf theorem (see, for example, [6]) states that the solution curve $z(t)$ exists for $t \in[0, M)$ for a certain positive constant $M$.

Suppose $T<M$ be the smallest value of $T$ such that $z(T)$ is on the boundary of the closed ball $B(z, \rho)$. Then

$$
\|z-z(T)\|_{\infty} \leqq \int_{0}^{T}\left\|\frac{d z(t)}{d t}\right\|_{\infty} d t<k\|f(z)\|_{\infty},
$$

where $k$ is defined by

$$
k=\max _{z^{\prime} \in B}\left\|f^{\prime}\left(z^{\prime}\right)^{-1}\right\|_{\infty} .
$$

This result was used in Refs. [3,7]. In fact, $z(t)$ satisfies

$$
\frac{d f(z(t))}{d t}=-f(z(t)) \text { with } z(0)=z .
$$

Thus,

$$
f(z(t))=f(z) e^{-t}
$$

holds. Hence, we have

$$
\begin{aligned}
\left\|\frac{d z(t)}{d t}\right\|_{\infty} & \leqq\left\|f^{\prime}(z(t))^{-1}\right\|_{\infty}\|f(z(t))\|_{\infty} \\
& \leqq k\|f(z)\|_{\infty} e^{-t}
\end{aligned}
$$

which gives

$$
\begin{aligned}
\int_{0}^{T}\left\|\frac{d z(t)}{d t}\right\|_{\infty} d t & \leqq k\|f(z)\|_{\infty}\left(1-e^{-T}\right) \\
& <k\|f(z)\|_{\infty} .
\end{aligned}
$$

Furthermore, $f(z(t))=f(z) e^{-t}$ implies $z(t)$ starting with an interior point remains to be an interior point for $t \in[0, M)$.

We note that for $z^{\prime} \in B(z, \rho)$

$$
\left\|f^{\prime}(z)^{-1}\left(f^{\prime}(z)-f^{\prime}\left(z^{\prime}\right)\right)\right\|_{\infty} \leqq \omega\left\|z-z^{\prime}\right\|_{\infty}
$$

holds. We note also that (14) implies

$$
\omega \rho<1 \text {. }
$$

Thus, from (34), it follows that

$$
\begin{aligned}
k & =\max _{z^{\prime} \in B}\left\|f^{\prime}\left(z^{\prime}\right)^{-1}\right\|_{\infty} \\
& \leqq \max _{z^{\prime} \in B} \frac{\left\|f^{\prime}(z)^{-1}\right\|_{\infty}}{1-\left\|I-f^{\prime}(z)^{-1} f^{\prime}\left(z^{\prime}\right)\right\|_{\infty}} \\
& =\max _{z^{\prime} \in B} \frac{\left\|f^{\prime}(z)^{-1}\right\|_{\infty}}{1-\left\|f^{\prime}(z)^{-1}\left(f^{\prime}(z)-f^{\prime}\left(z^{\prime}\right)\right)\right\|_{\infty}} \\
& \leqq \max _{z^{\prime} \in B} \frac{\left\|f^{\prime}(z)^{-1}\right\|_{\infty}}{1-\omega\left\|z-z^{\prime}\right\|_{\infty}}
\end{aligned}
$$

$$
\leqq \frac{\left\|f^{\prime}(z)^{-1}\right\|_{\infty}}{1-\omega \rho}
$$

Therefore, we have

$$
\begin{aligned}
k\|f(z)\|_{\infty} & \leqq \frac{\left\|f^{\prime}(z)^{-1}\right\|_{\infty}\|f(z)\|_{\infty}}{1-\omega \rho} \\
& \leqq \frac{\alpha}{1-\omega \rho} .
\end{aligned}
$$

On the other hand, we can show the following inequality

$$
\frac{\alpha}{1-\omega \rho} \leqq \rho
$$

In fact, since $4 \alpha \omega \leqq 1$, we have the inequality

$$
1-2 \alpha \omega \leqq \sqrt{1-3 \alpha \omega}
$$

which implies the inequality

$$
\frac{\alpha}{\sqrt{1-3 \alpha \omega}} \leqq \frac{1-\sqrt{1-3 \alpha \omega}}{\omega}
$$

which is equivalent to the inequality (38).

The inequalities (28), (37) and (38) imply

$$
\|z-z(T)\|_{\infty}<\rho
$$

which contradicts the fact that $z(T)$ is on the boundary of $B(z, \rho)$. Therefore, there exists no such $T$ and the solution curve is contained in the interior of the ball $B(z, \rho)$. There is no singularity of the right hand side of $(27)$ in $B(z, \rho)$. By the elementary theory of differential equation on extending solutions (see, for instance, [8]), the solution can be prolonged to the interval $[0, \infty)$, i.e., $M=\infty$ and it converges to $z^{*}$ as $t$ tends to $\infty$. In fact, let $z^{* *}$ be a point in the limit set of the solution curve, which is obviously contained in the closed ball $B(z, \rho)$. Then $z^{* *}$ is a solution of (6) by (31). By the uniqueness of the solution of $(6)$ in the closed ball $B(z, \rho)$, it is identical to $z^{*}$. Therefore, the solution curve converges to $z^{*}$ as $t$ tends to $\infty$.

Since the solution curve is contained in the feasible set, the limit point $z^{*}$ is also a feasible point.

(QED)

\section{Numerical Examples}

In this section, let us present numerical examples. For executing verified computation, we have used MATLAB on Windows XP over a personal computer having Core 2 Duo $1.2 \mathrm{GHz}$ Intel processor.

A verification function is programmed based on the rounding mode controlled numerical verification method proposed in Ref. [9].

Example 1 Let us consider the following problem:

Maximize $c^{t} x$, subject to $A x \leqq b$ and $x \geqq 0$,

where $c^{t}=(3,2)$,

$$
A=\left(\begin{array}{cc}
-1 & 3 \\
1 & 1 \\
2 & -1
\end{array}\right)
$$

and $b^{t}=(12,8,10)$. It is known that the optimal solution 
is $x=(6,2)^{t}$. In this case, we have an interior point

$$
\begin{aligned}
& x=\left(\begin{array}{l}
5.999999999999999 \\
2.000000000000000
\end{array}\right), \\
& y=\left(\begin{array}{l}
4.166666666666667 \times 10^{-017} \\
2.333333333333334 \\
3.333333333333334 \times 10^{-1}
\end{array}\right) .
\end{aligned}
$$

For this point, we have

$$
\alpha \omega<7.93 \times 10^{-14} .
$$

Thus, there exists an optimum solution of (42) in the ball centered at $z=\left(x^{t}, y^{t}\right)^{t}$ with a radius

$$
\rho=5.14 \times 10^{-15} \text {. }
$$

Further, the objective value is included in [22.00000000000000,22.00000000000001]. These results are consistent with the exact solution $x=(6,2)^{t}$.

Example 2 Next, let us consider the following simple linear programming problem:

Maximize $c^{t} x$, subject to $A x \leqq b$ and $x \geqq 0$,

where $c^{t}=(300,300,500)$,

$$
A=\left(\begin{array}{ccc}
150 & 100 & 100 \\
1 & 2 & 1 \\
0 & 0 & 150
\end{array}\right)
$$

and $b^{t}=(3000,40,1200)$. In this case, we have a feasible solution

$$
\begin{aligned}
& x=\left(\begin{array}{l}
5.9999999999999973 \\
13.000000000000004 \\
8.0000000000000000
\end{array}\right), \\
& y=\left(\begin{array}{l}
1.5000000000000000 \\
75.000000000000000 \\
1.8333333333333335
\end{array}\right) .
\end{aligned}
$$

For this feasible point, we have

$$
\alpha \omega<1.64 \times 10^{-11} .
$$

Thus, there exists an optimum solution of (46) in the ball centered at $z=\left(x^{t}, y^{t}\right)^{t}$ with a radius

$$
\rho=1.45 \times 10^{-13} \text {. }
$$

Example 3 In this example, we shall consider the following problem with $n=2 m$.

$$
\text { Maximize } c^{t} x \text {, subject to } A x \leqq b \text { and } x \geqq 0,
$$

where

$$
c=10 c_{r}, A=10 E+5 A_{r}, b=100 b_{r}
$$

Here, $c_{r} \in \mathbb{F}^{n}$ is a pseudo-random vector whose elements are distributed uniformly in $[0,1], E \in \mathbb{F}^{m \times n}$ is a con-

\begin{tabular}{|c|c|c|c|c|}
\hline$m+n$ & $\alpha \omega$ & $\rho$ & $t_{a}[\mathrm{sec}]$ & $t_{v}[\mathrm{sec}]$ \\
\hline 300 & $3.78 \times 10^{-6}$ & $3.4 \times 10^{-12}$ & 0.30 & 0.29 \\
\hline 600 & $1.69 \times 10^{-5}$ & $5.0 \times 10^{-12}$ & 0.79 & 2.17 \\
\hline 900 & $4.22 \times 10^{-4}$ & $2.9 \times 10^{-11}$ & 1.9 & 7.4 \\
\hline 1200 & 0.0049 & $7.3 \times 10^{-11}$ & 3.9 & 17.4 \\
\hline 1500 & 0.083 & $3.8 \times 10^{-10}$ & 7.2 & 33.4 \\
\hline 1800 & $2.91 \times 10^{-4}$ & $1.8 \times 10^{-11}$ & 13 & 59 \\
\hline 2100 & 0.189 & $4.7 \times 10^{-10}$ & 21 & 91 \\
\hline 2400 & 0.051 & $2.1 \times 10^{-10}$ & 37 & 137 \\
\hline 2700 & 0.029 & $1.4 \times 10^{-10}$ & 54 & 184 \\
\hline 3000 & 0.011 & $8.3 \times 10^{-11}$ & 72 & 270 \\
\hline
\end{tabular}
stant matrix whose elements are all one, $A_{r} \in \mathbb{F}^{m \times n}$ is a pseudo-random matrix whose elements are distributed according to the normal distribution with the mean zero and the variation one, and $b_{r} \in \mathbb{F}^{m}$ is a pseudo-random vector whose elements are distributed uniformly in $[0,1]$.

We examine the cases of $m=100,200,300,400,500$, $600,700,800$ and $m=900$. We solve the problem on $M A T L A B$. The result is summarized in Table 1. In this table, $t_{a}$ is time for computing an approximate optimum solution and $t_{b}$ that for numerical verification.

In this numerical example, a computational cost to include optimum point is about 4 times than that for getting an approximate optimum solution.

\section{Acknowledgments}

This research is supported by the Grant-in-Aid for Specially Promoted Research from the MEXT, Japan: "Establishment of Verified Numerical Computation", (No. 17002012). The authors express their sincere thanks to the referees for their valuable comments on this article.

\section{References}

[1] C. Jansson, Rigorous lower and upper bounds in linear programming, SIAM J. Optim., 14(3) (2004), 914-935.

[2] K. Tanabe, A posteriori error estimate for an approximate solution of a general linear programming, in: New Methods for Linear Programming 2, The Institute of Statistical Mathematics Cooperative Research Report 10, pp.118-120, 1988.

[3] K. Tanabe, A geometric method in nonlinear programming, J. Optim. Theory Appl., 30 (1980), 181-210.

[4] K. Tanabe, Complementarity-enforcing centered newton method for mathematical programming: global method, in: New Methods for Linear Programming, The Institute of Statistical Mathematics Cooperative Research Report 5, pp.118144, 1987.

[5] K. Tanabe, Centered Newton method for mathematical programming, in: System Modeling and Optimization, M. Iri and K.Yajima eds., pp.197-208, Springer-Verlag, Berlin, 1988.

[6] E. Zeitler, Nonlinear Functional Analysis and Its Applications: Part I Fixed Point Theorems, Springer-Verlag, New York, 1986, p.78.

[7] K. Tanabe, Continuous Newton-Raphson method for solving an underdetermined system of nonlinear equations, Nonlinear Anal. T.M.A., 3 (1979), 495-503.

[8] M. W. Hirsch and S. Smale, Differential Equations, Dynamical Systems, and Linear Algebra, Academic Press, London, 1974, p. 171 .

[9] S. Oishi and S.M. Rump, Fast verification of solutions of matrix equations, Numer. Math., 90 (4) (2002), 755-773. 